\title{
SPATIAL SOUND REPRODUCTION SYSTEMS USING HIGHER ORDER LOUDSPEAKERS
}

\author{
Mark A. Poletti \\ Industrial Research Ltd, \\ PO Box 31-310, Lower Hutt \\ New Zealand
}

\author{
Thushara D. Abhayapala
}

\author{
ANU College of Engineering \& Computer \\ Science, The Australian National University \\ Canberra ACT 0200, Australia
}

\begin{abstract}
Sound reproduction systems aim to produce a desired sound field over a region of space. At high frequencies, the number of loudspeakers required is prohibitive. This paper shows that the use of Nth order loudspeakers, in which each loudspeaker produces polar responses up to $\cos (N \phi)$ and $\sin (N \phi)$, produces accurate reproduction over $N$ times the area of a first order array and can largely eliminate any exterior field. This allows a significant reduction in the number of loudspeaker units, at the expense of increased complexity in each loudspeaker unit.
\end{abstract}

Index Terms - Sound, surround, reproduction, loudspeakers, sources

\section{INTRODUCTION}

Sound reproduction systems aim to reproduce an arbitrary desired sound field within a region of space. The desired sound field may be generated using the Kirchhoff-Helmholtz (K-H) integral [1], or cylindrical or spherical harmonic decompositions (higher order Ambisonics) [2,3].

The accuracy of sound reproduction is governed by the wavelength and the size of the region over which reproduction is required. For wave number $k$ and reproduction radius $r$ the number of required loudspeakers in the $2 \mathrm{D}$ case is given approximately by [2]

$$
L \approx 2 k r+1 \text {. }
$$

Hence, large numbers of loudspeakers are required for the reproduction of high frequencies over significant areas. For example, reproduction over $0.1 \mathrm{~m}$ radius at $16 \mathrm{kHz}$ requires 60 loudspeakers. In the $3 \mathrm{D}$ case the required number of loudspeakers is significantly higher [3].

A further limitation of reproduction in rooms is that the loudspeakers produce a reverberant field which corrupts the sound field within the array [4]. This reverberant field can be cancelled using calibration and pre-processing but such techniques require accurate measurement of acoustic transfer functions and significant computing power [3,5-7]. If, however, loudspeakers with omnidirectional and radial dipole directivities are used, it is possible to produce a sound field within the loudspeaker array and no exterior field, by using the K-H integral [1]. Exterior cancellation is possible below the Nyquist frequency of the array, where the transducers are a half wavelength apart which, in the 2D case and for a loudspeaker radius $r_{L}$, and speed of sound $c$, is [8]

$$
f_{1}=c(L-1) /\left(4 \pi r_{L}\right) .
$$

We use the subscript 1 to denote the fact that the loudspeakers have first order polar responses.

At frequencies at or above Nyquist, the K-H approach fails and a nonzero exterior sound field is produced. In this case fixed-directivity speakers can reduce the exterior field $[4,9,10]$. The K-H approach can, however, be made more accurate near the Nyquist frequency by including tangential dipoles in each loudspeaker response [8]. This suggests that higher order variable polar responses may produce further improvements in sound reproduction. In this paper we show that this is indeed the case. We present the 2D cylindrical harmonic description of higher order sound sources and derive the required source amplitudes for producing a desired sound field. It is shown through simulations that a circular array of $N$ th order loudspeakers produces a Nyquist frequency of $N$ times that in (2). Hence, the reproduction area, or equivalently, the bandwidth of accurate reconstruction over a specified area, is increased by a factor $N$.

\section{THEORY}

The interior solution to the wave equation at a point $\vec{r}=(r, \phi)$ in the simple case of height invariance has the general form [11]

$$
p(r, \phi, k)=\sum_{m=-\infty}^{\infty} J_{m}(k r) A_{m}(k) e^{i m \phi}
$$

where $J_{m}($.$) is the m t h$ cylindrical Bessel function, and $A_{m}$ the mth expansion coefficient. Due to the properties of the Bessel function, this expansion can be truncated to order $M \approx k r$ for a radius $r$ and wavenumber $k$ [2]. From (1), with 
$L$ loudspeakers, the desired field can be reproduced up to mode order

$$
M=(L-1) / 2 .
$$

Equation (3) represents a desired sound field we wish to produce within a circular array of $L$ loudspeakers positioned at $\vec{r}_{l}=\left(r_{L}, \phi_{l}\right), l \in[1, L]$.

In a simple zeroth order sound reproduction system, each loudspeaker is a monopole [8], with expansion [11]

$$
H_{0}^{(1)}\left(k r_{0}\right)= \begin{cases}\sum_{m=-\infty}^{\infty} J_{m}(k r) H_{m}^{(1)}\left(k r_{l}\right) e^{i m\left(\phi-\phi_{l}\right)}, & r<r_{l} \\ \sum_{m=-\infty}^{\infty} J_{m}\left(k r_{l}\right) H_{m}^{(1)}(k r) e^{i m\left(\phi-\phi_{l}\right)}, r>r_{l}\end{cases}
$$

where $H_{m}^{(1)}($.$) is the m t h$ cylindrical Hankel function of the first kind and $r_{0}=\left\|\vec{r}_{-} \vec{r}_{l}\right\|$. We assume a single frequency sound field with a time dependency $\exp (-i \omega t)$, which produces outward propagating wave fronts.

Zeroth order sources can produce a desired interior field, but first order sources are required to independently control the exterior field. Here we consider the more general case of Nth order sources, which are represented as

$$
p_{n}(r, \phi)=H_{n}^{(1)}\left(k r_{0}\right) e^{i n \beta_{l}}
$$

where $\beta_{l}$ is the angle measured from the field point to the source vector $\vec{r}_{l}$ (figure 1 ).

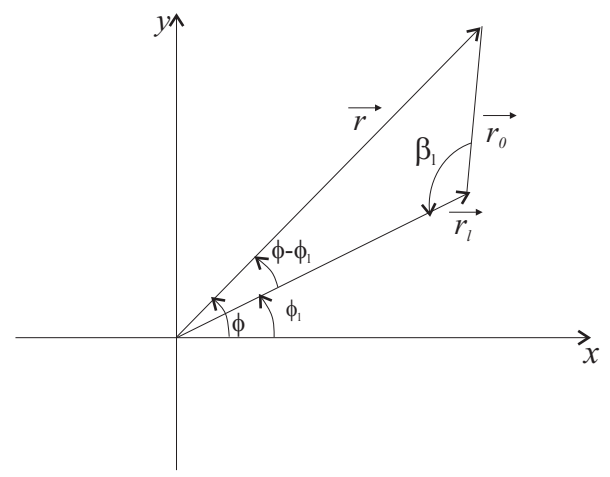

Fig. 1: Higher order source geometry for source vector $\vec{r}_{l}$ and field vector $\vec{r}$

The $n$th order sound field (6) can be expressed in a similar manner to (5) using the cylindrical addition theorem [12].

$$
p_{n}(r, \phi)=\left\{\begin{array}{ll}
\sum_{m=-\infty}^{\infty} J_{m}(k r) H_{m+n}\left(k r_{l}\right) e^{i m\left(\phi-\phi_{l}\right)}, & r<r_{l} . \\
\sum_{m=-\infty}^{\infty} J_{m+n}\left(k r_{l}\right) H_{m}(k r) e^{i m\left(\phi-\phi_{l}\right)}, r>r_{l}
\end{array} .\right.
$$

A combination of order $n=N$ and $n=-N$ sources has a farfield polar response which is a combination of $\cos (N \phi)$ and $\sin (N \phi)$ terms. A loudspeaker which produces a general Nth order source consists of a sum of source orders $n \in[-N, N]$ with weights $w_{n, l}$.

$$
p_{N l}\left(r, \phi, r_{L}, \phi_{l}\right)=\sum_{n=-N}^{N} w_{n, l} H_{n}\left(k\left\|\vec{r}-\vec{r}_{l}\right\|\right) e^{i n \beta_{l}} .
$$

An array of $L$ such sources then produces the sound field

$$
\hat{p}(r, \phi)=\sum_{l=1}^{L} \sum_{n=-N}^{N} w_{n, l} H_{n}\left(k\left\|\vec{r}-\vec{r}_{l}\right\|\right) e^{i n \beta_{l}} .
$$

which must closely approximate the desired field in (3).

\section{SOLUTION}

Substituting equation (7) into (9) and equating with (3), then multiplying each side by $\exp (-i v \phi)$ for integers $v$ and integrating over $\phi$ yields, for each mode $m$, the interior mode matching equation

$$
\sum_{n=-N}^{N} H_{m+n}\left(k r_{L}\right) \sum_{l=1}^{L} w_{n, l} e^{-i m \phi_{l}}=A_{m}, m \in[-M, M] .
$$

A similar process produces, for the exterior sound field

$$
\sum_{n=-N}^{N} J_{m+n}\left(k r_{L}\right) \sum_{l=1}^{L} w_{n, l} e^{-i m \phi_{l}}=0, m \in[-M, M] .
$$

The set of equations (10) and (11) may be put in matrix form

$$
\mathbf{H} w=d,
$$

where $\mathbf{H}$ is a $(4 M+2)$ by $(2 N+1) L$ matrix, $w$ is a $(2 N+1) L$ by 1 vector of weights $w_{n, l}$ and $d$ is a $(4 M+2)$ by 1 vector containing the $2 M+1$ desired sound field coefficients, and $2 M+1$ zeros.

This matrix equation can be solved for the higher order source weights when $4 M+2$ is less than $(2 N+1) L$ (the underdetermined case) using a minimum energy criterion $[2,3]$. This suggests that the maximum number of modes that can be reproduced in the interior of the array is $M=(N+1 / 2) L / 2-1 / 2$. However, in the first order case the mode order is given by (4) which is inconsistent with the above for $N=1$. Since exterior cancellation is only possible for first order sources, we infer that the maximum mode order that can be controlled for interior/exterior control is

$$
M(N)=(N L-1) / 2, N>0
$$

which is consistent with (4). The maximum region of reproduction is, with this assumption (and using the $M \approx k r$ equivalence),

$$
r_{N}(f)=c(L N-1) /(4 \pi f) .
$$

The maximum frequency at which the reproduction system can produce accurate reconstruction out to a radius $r_{L}$ and cancel the exterior field is the Nth order Nyquist frequency

$$
f_{N}=c(L N-1) /\left(4 \pi r_{L}\right) .
$$

This suggests that an Nth order array is able to produce $N$ times the performance of a first order array. 


\section{SIMULATION RESULTS}

To investigate the performance improvement of an Nth order loudspeaker array we developed Matlab simulations of a circular array containing $L=15$ higher order sources. The array radius is $3 \mathrm{~m}$ and, from (2), this produces a first order array Nyquist frequency of $126 \mathrm{~Hz}$. We aim to reproduce the sound field due to a line source, as in equation (5), at a source radius of $6 \mathrm{~m}$ and a worst-case source angle of 36 degrees, half way between adjacent loudspeakers.

Figure 2 shows the sound field produced at a frequency of $125 \mathrm{~Hz}$, at the Nyquist frequency of the array, for $N=1$, the $\mathrm{K}-\mathrm{H}$ case. The circle shows the maximum radius of reproduction from (14). Reproduction is accurate over the interior region, and the exterior sound field is small.

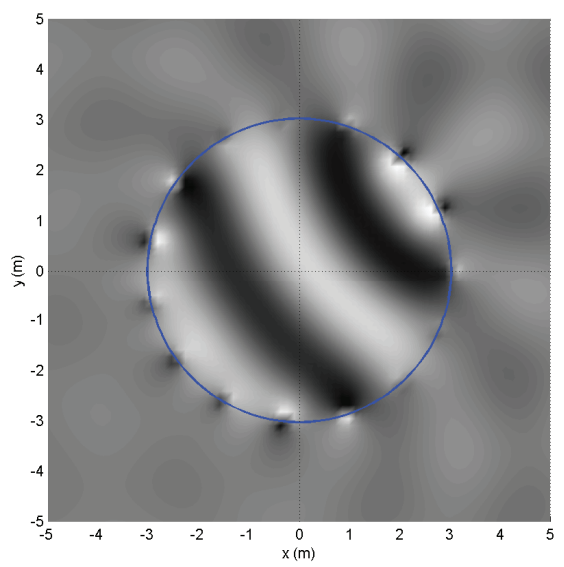

Fig. 2: Sound field for $N=1$, frequency $125 \mathrm{~Hz}$

At $350 \mathrm{~Hz}$, well above the Nyquist frequency of the firstorder array, the maximum radius of reproduction is $1.08 \mathrm{~m}$, and the sound field, shown in figure 3 , is inaccurate for radii greater than this value. The loudspeakers are greater than half a wavelength apart and are therefore unable to generate a cancelled exterior field.

The reproduction error relative to the desired pressure at the origin,

$$
\mathcal{E}(r, \phi)=\left\{\begin{array}{cc}
|\hat{p}(r, \phi)-p(r, \phi)| /|p(0)|, & r<r_{L} \\
|\hat{p}(r, \phi)| /|p(0)|, & r>r_{L}
\end{array},\right.
$$

for the sound field in figure 3 is shown in figure 4 . The field is incorrect for radii greater than $1.08 \mathrm{~m}$, and the exterior field is no longer small. Figure 5 shows the field at $350 \mathrm{~Hz}$ using a source order $N=3$, for which, from (15), the array Nyquist frequency is $400 \mathrm{~Hz}$. The field is now accurate out to the loudspeaker radius and the exterior field is small.

The reproduction error is shown in figure 6 . The region of accurate reproduction is three times that of the first order array, supporting equation (14). The exterior field is small, but as the source frequency approaches Nyquist, the exterior field begins to increase.

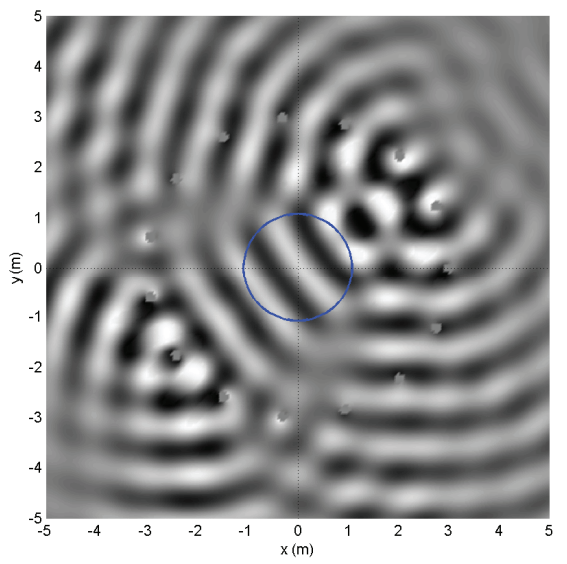

Fig. 3: Sound field for $N=1$, frequency $350 \mathrm{~Hz}$

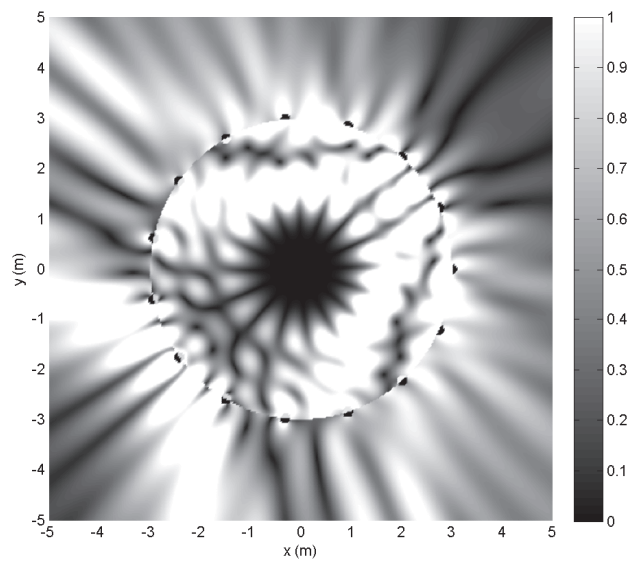

Fig. 4: Reproduction error (16), $N=1$, frequency $350 \mathrm{~Hz}$

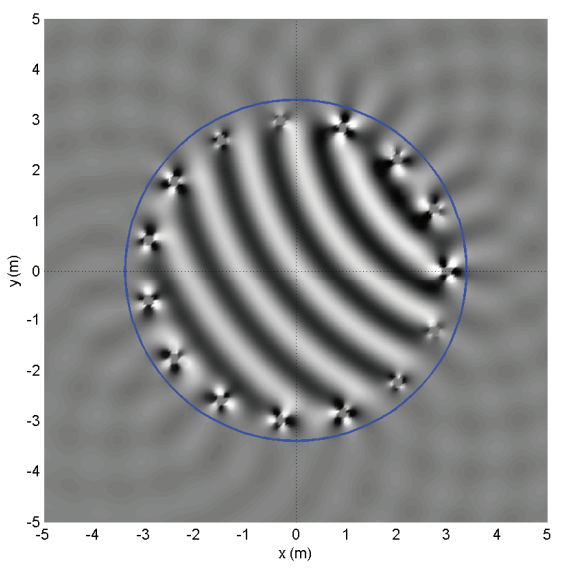

Fig. 5: Sound field for $N=3$, frequency $350 \mathrm{~Hz}$ 


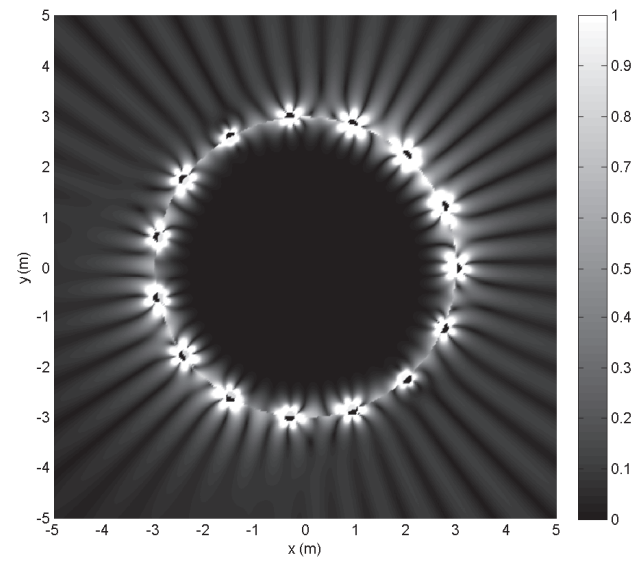

Fig. 6: Reproduction error (16), $N=3$, frequency $350 \mathrm{~Hz}$

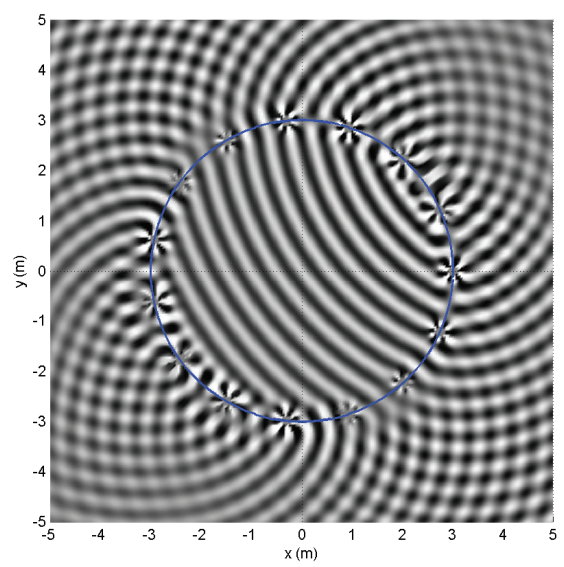

Fig. 7: Sound field for $N=6$, frequency $800 \mathrm{~Hz}$

This is shown in figure 7 for a $6^{\text {th }}$ order array at a frequency of $800 \mathrm{~Hz}$, close to the Nyquist frequency of the array (803 $\mathrm{Hz}$ ). The interior field is accurate within the array, confirming again the $\mathrm{N}$-fold increase in reproduction radius. The exterior field is now significant, but rapidly reduces in amplitude for frequencies below $800 \mathrm{~Hz}$

\section{CONCLUSION}

This paper has considered the use of an array of higher order sound sources for sound field reproduction, in the 2D case, which can in practice be approximated using monopole sources $[13,14]$. It has been demonstrated by simulation that an Nth order array - capable of radiating polar responses up to and including $\cos (N \phi)$ and $\sin (N \phi)-$ is able to extend the reproduction region, or the frequency range, by a factor $N$, while significantly reducing the exterior sound field. This suggests that sound reproduction can be carried out using
$1 /$ Nth of the number of simple loudspeakers, if those loudspeakers are able to produce all responses up to Nth order.

\section{REFERENCES}

[1] A. J. Berkhout, D. de Vries and P. Vogel, "Acoustic control by wave field synthesis," J. Acoust. Soc. Am., 93 (5), pp 2764-2778, 1993

[2] D. B. Ward and T. D. Abhayapala, "Reproduction of a planewave sound field using an array of loudspeakers," IEEE Trans. Speech Audio Proc., 9 (6), pp 697 - 707, 2001

[3] M. A. Poletti, "Three dimensional surround sound systems based on spherical harmonics," J. Audio Engin. Soc., 53 (11), pp 1004-1025, November 2005

[4] M. A. Poletti, F. M. Fazi and P. A. Nelson, "Sound-field reproduction systems using fixed-directivity loudspeakers," $J$. Acoust. Soc. Am. 127 (6), pp 3590-3601, 2010

[5] P-A Gauthier, A. Berry and W. Woszczyk, "Sound-field reproduction in-room using optimal control techniques: Simulations in the frequency domain," J. Acoust. Soc. Am., 117 (2), pp 662-678, February 2005

[6] S. Spors, H. Buchner, R. Rabenstein and W. Herbordt, "Active listening room compensation for massive multichannel sound reproduction systems using wave-domain adaptive filtering," $J$. Acoust. Soc. Am., 122 (1), pp 354-369, July 2007

[7] T. Betlehem and T. Abhayapala, "Theory and design of sound field reproduction in reverberant rooms," J. Acoust. Soc. Am., 117, Pt. 1, pp 2100-2111, April 2005

[8] M. A. Poletti and T. D. Abhayapala, "Interior and exterior sound field control using general two dimensional first-order sources," J. Acoust. Soc. Am., 129 (1), pp 234-244, January 2011

[9] D. de Vries, "Sound reinforcement by wavefield synthesis: adaptation of the synthesis operator to the loudspeaker directivity characteristics," J. Audio Engin. Soc., 44 (12), pp 1120-1131, 1996

[10] J. Ahrens and S. Spors, "Sound field reproduction employing non-omnidirectional loudspeakers," Audio Engin. Soc. $126^{\text {th }}$ Conv., Munich, Germany, 2009

[11] E. G. Williams, Fourier Acoustics, Academic Press, San Deigo, 1999, p. $115-125$, p. 266

[12] G. N. Watson, A Treatise on the Theory of Bessel Functions, 2nd Edition, Cambridge University Press, 1995, p. 361

[13] R. Nicol and M. Emerit, "3D-Sound Reproduction over an Extensive Listening Area: A Hybrid Method Derived from Holophony and Ambisonic," Audio Engin. Soc. 16th Int. Conf., Rovaniemi, Finland (1999).

[14] J. Ahrens and S. Spors, "An analytical approach to sound field reproduction using circular and spherical loudspeaker distributions," Acta Acustica, Acustica, 94, pp 988-999, 2008 